Hahn/Kaufmann (Hrsg.)

Handbuch Industrielles Beschaffungsmanagement 
Dietger Hahn/Lutz Kaufmann (Hrsg.)

\section{Handbuch \\ Industrielles \\ Beschaffungsmanagement}

Internationale Konzepte - Innovative Instrumente - Aktuelle Praxisbeispiele

2., überarbeitete und erweiterte Auflage

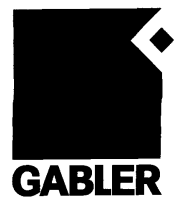


Bibliografische Information Der Deutschen Bibliothek

Die Deutsche Bibliothek verzeichnet diese Publikation in der Deutschen Nationalbibliografie;

detaillierte bibliografische Daten sind im Internet über <http://dnb.ddb.de> abrufbar.

Univ.-Prof. Dr. Dr. h.c. mult. Dietger Hahn

Institut für Unternehmensplanung (IUP), Gießen/Berlin

Licher Str. 62, 35394 Gießen

Telefon: 0641 / 47640

Telefax: 0641 / 493507

Univ.-Prof. Dr. Lutz Kaufmann MBA

WHU Wissenschaftliche Hochschule für Unternehmensführung (Otto-Beisheim-Hochschule)

Herbert-Quandt-Stiftungslehrstuhl für Betriebswirtschaftslehre,

insbesondere Internationales Management

Burgplatz 2, 56179 Vallendar

Telefon: 0261 / $6509-320$

Telefax: 0261 / 6509 - 329

email: kaufmann@whu.edu

Internet: www.whu.edu/intman/

1. Auflage April 1999

2. Auflage Oktober 2002

Alle Rechte vorbehalten

(C) Springer Fachmedien Wiesbaden 2002

Ursprünglich erschienen bei Betriebswirtschaftlicher Verlag Dr. Th. Gabler GmbH, Wiesbaden 2002 Softcover reprint of the hardcover 2nd edition 2002

Lektorat: Barbara Roscher / Jutta Hinrichsen

www.gabler.de

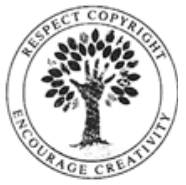

Das Werk einschließlich aller seiner Teile ist urheberrechtlich geschützt. Jede Verwertung außerhalb der engen Grenzen des Urheberrechtsgesetzes ist ohne Zustimmung des Verlags unzulässig und strafbar. Das gilt insbesondere für Vervielfältigungen, Übersetzungen, Mikroverfilmungen und die Einspeicherung und Verarbeitung in elektronischen Systemen.

Die Wiedergabe von Gebrauchsnamen, Handelsnamen, Warenbezeichnungen usw. in diesem Werk berechtigt auch ohne besondere Kennzeichnung nicht zu der Annahme, dass solche Namen im Sinne der Warenzeichen- und Markenschutz-Gesetzgebung als frei zu betrachten wären und daher von jedermann benutzt werden dürften.

Umschlaggestaltung: Regine Zimmer, Dipl.-Designerin, Wiesbaden

Gedruckt auf säurefreiem und chlorfrei gebleichtem Papier

ISBN 978-3-663-01583-3

ISBN 978-3-663-01582-6 (eBook)

DOI $10.1007 / 978-3-663-01582-6$ 


\section{Vorwort der Herausgeber zur zweiten Auflage}

Die äußerst positive Resonanz auf die erste Auflage und die damit verbundene Verbreitung haben uns schneller als erwartet die Möglichkeit gegeben, dieses Handbuch zu überarbeiten. Die inhaltliche Struktur ist dabei unverändert geblieben, ebenso wie die bewährte Mischung aus wissenschaftlichen und praxisorientierten Beiträgen. Wir freuen uns darüber, neben Europa und den USA nun auch einen ersten Beitrag aus Asien präsentieren zu können. Somit spiegelt das Handbuch jetzt noch stärker die regionalen Schwerpunkte der weltweiten Beschaffungsaktivitäten vieler deutscher Unternehmen wider.

In der Zeit seit der Veröffentlichung der ersten Auflage hat das Beschaffungsmanagement eine weitere Professionalisierung in der Praxis und eine theoretisch und methodisch fundiertere Behandlung in der Wissenschaft erfahren. Das betrifft insbesondere, aber nicht nur, die Unterstützung der Beschaffung durch das Internet, beispielsweise durch elektronische Auktionen. Sämtliche überarbeiteten Artikel berücksichtigen solche aktuellen Entwicklungen. Wir hoffen daher, daß auch die Leser dieser Auflage mit uns der Meinung sind, das Handbuch repräsentiere wieder einen wesentlichen Beitrag zum „State of the Art“ im Beschaffungsmanagement.

Als Wissenschaftler mit praktischen Wurzeln wissen wir um die Zusatzbelastung, die die Übernahme eines Beitrages für ein solches Werk mit sich bringt, gerade für Praktiker. Daher danken wir sehr herzlich allen Autoren für ihre Zeit und ihr Engagement beim Verfassen neuer bzw. Überarbeiten existierender Beiträge. Unsere Teams an unseren Lehrstühlen in Gießen und Vallendar/Koblenz haben uns oft bei der ,technischen“ Erstellung der zweiten Auflage engagiert unterstützt - auch ihnen sei an dieser Stelle herzlich gedankt. Unser ganz besonderer Dank gebührt dabei erneut Thomas Germer, der nicht nur selbst einen Artikel in diesem Buch mit verfasst hat, sondern auch mit großer Hingabe die komplette grafische und formale Überarbeitung dieser Auflage über den gesamten Projektverlauf hinweg maßgeblich gesteuert und umgesetzt hat. 


\section{Vorwort der Herausgeber zur ersten Auflage}

Der Aufgabenkomplex der Beschaffung hat in den vergangenen Jahren in den meisten Industrieunternehmen eine deutliche Aufwertung erfahren. Dies hat vielfältige Ursachen. Häufig wurden in den Unternehmen die Leistungstiefen mit dem Ziel reduziert, die Ressourcen auf Kernbereiche zu konzentrieren. Dadurch bekommt die Beschaffung eine ganz wesentliche Bedeutung für das Unternehmensergebnis. Daß ein optimiertes Beschaffungsmanagement zu einem wettbewerbsentscheidenden Faktor avancieren kann, hat nicht zuletzt der viel zitierte Lopéz-Effekt in der Automobilindustrie gezeigt. Doch auch in Branchen mit anderen Marktlagen als in der Automobilindustrie der frühen neunziger Jahre kann ein sehr erfolgreiches Beschaffungsmanagement betrieben werden.

Verbände und die Wissenschaft haben die Managementinnovationen in der Beschaffung bereits seit geraumer Zeit angeregt und systematisierend begleitet. An den deutschen Hochschulen allerdings hat das Supply Chain Management und damit die Beschaffung noch bei weitem nicht den Stellenwert wie in der Unternehmenspraxis. Oft muß Beschaffungsmanagement hier noch ehrenamtlich vertreten werden.

Mit dem Handbuch Industrielles Beschaffungsmanagement wollen wir ein Grundlagenwerk vorlegen, das den „State of the Art“ vorstellt. Wir waren der Meinung, daß es dazu erforderlich sei, hochkarätige Autoren aus Wissenschaft und Praxis zusammenzubringen, und zwar aus Europa und aus den USA. Daher ist jeder der sechs Handbuchteile so konzipiert, daß darin neben deutschsprachigen wissenschaftlichen Beiträgen jeweils mindestens ein internationaler Beitrag und mindestens zwei aktuelle Praxisbeispiele aus unterschiedlichen Branchen zu finden sind.

Der erste Handbuchteil ist den Grundlagen modernen Beschaffungsmanagements gewidmet. Im zweiten Teil werden diese Grundlagen verfeinert, indem mögliche strategische Ausrichtungen für die industrielle Beschaffung, z.B. im Hinblick auf die Festlegung der optimalen Lieferantenzahl, die Lieferantenentwicklung und das Global Sourcing, aufgezeigt werden. Der dritte Teil beschäftigt sich mit dem Zusammenspiel von Beschaffung und funktionalen Unternehmensbereichen wie beispielsweise Forschung und Entwicklung, Produktion, Logistik und Qualitätsmanagement. Um die Beschaffungsaktivitäten in ihrem Zusammenspiel mit anderen Bereichen optimal analysieren, planen, implementieren und kontrollieren zu können, sind innovative Instrumente erforderlich. Der vierte Teil verdeutlicht solche Instrumente und gibt zahlreiche Beispiele dafür, wie diese in der Praxis erfolgreich eingesetzt werden können. Modernes Beschaffungsmanagement erfordert nicht nur eine klare strategische Ausrichtung: Umgesetzt werden Beschaffungsstrategien nach wie vor im Tagesgeschäft, und für dieses ist eine strategiekonforme Gestaltung der Organisation sowie der Informations- und Kommunikationssysteme unverzichtbar. Im fünften Teil werden hierfür Alternativen und Anwendungsbeispiele vorgestellt. Der sechste Teil des Handbuches trägt der Tatsache Rechnung, daß erfolgreiches Beschaffungsmanagement nicht nur strategiegeleitet, gut organisiert und 
informationstechnologisch up to date sein muß, sondern vor allem von dem „Können, Wollen und Dürfen“ der Mitarbeiter abhängt. Diesem sechsten Teil können Impulse für ein erfolgreiches Personalmanagement für die industrielle Beschaffung entnommen werden. Im Ausblick werden Thesen zur Zukunft des Beschaffungsmanagements in einem integrierten Supply Chain Management formuliert.

Das moderne Beschaffungsmanagement wird künftig integraler Bestandteil des Supply Chain Management sein, das die gesamten wertschöpfenden Aktivitäten von der Rohstoffquelle bis zum Endkonsumenten überspannt. Aus klassischen Interpretationen des Unternehmens können auch für diese Epoche Erklärungsmuster und Gestaltungshinweise abgeleitet werden: Interpretiert man das Unternehmen als Interessenzentrum, so erkennt man rasch die Bedeutung der Kundenorientierung, der Mitarbeitermotivation und -entwicklung sowie der Pflege partnerschaftlicher Lieferantenbeziehungen. Aus der Interpretation von Unternehmen als zielorientierte Aktionszentren läßt sich die Notwendigkeit ableiten, die Prozeßorientierung auf die gesamte Supply Chain auszudehnen. Die Sicht des Unternehmens als sozio-technisches System und in der Tradition der Systemtheorie stehende Ansätze, wie die Netzwerktheorie, haben Erklärungs- und Gestaltungskraft für die Vernetzung der organisatorischen Einheiten in und zwischen Unternehmen, die sich heute unter dem Einfluß der Informations- und Kommunikationstechnologie bis hin zu virtuellen Netzen vollzieht. Die Interpretation von Unternehmen schließlich als Vertragszentren ist Teil institutionenökonomischer Betrachtungen und kann auch für die Beziehungsregelung im juristischen Sinne herangezogen werden.

Das Buch richtet sich an Führungskräfte aus dem Bereich Supply Chain Management und insbesondere aus der Beschaffung, die sich neue Impulse für ihr Tagesgeschäft und für die Weiterentwicklung ihrer Beschaffungsstrategien holen möchten. Die wissenschaftlich tätige Leserschaft bekommt einen konzentrierten Überblick zum industriellen Beschaffungsmanagement und Anregungen für eigene Forschungsarbeiten. Zudem kann das Buch in der Lehre an Hochschulen und in Weiterbildungsseminaren eingesetzt werden.

Wir möchten uns zunächst sehr herzlich bei den zahlreichen Autoren aus Wissenschaft und Praxis bedanken: Einen Handbuchbeitrag zu verfassen bedeutet in aller Regel, über die ohnehin anstehenden Aufgaben hinaus, ein weiteres Projekt bearbeiten zu müssen. Diese Bereitschaft ist keineswegs selbstverständlich. Ohne diese Bereitschaft der Praktiker zum Dialog aber bliebe der Wissenschaft nur der Weg in den unrühmlichen Elfenbeinturm. Wir freuen uns deshalb sehr über unser internationales Autorenteam aus praxisnahen Wissenschaftlern und wissenschaftsnahen Praktikern. Schließlich bedanken wir uns sehr bei unseren studentischen Mitarbeitern am Lehrstuhl in Gießen: Auch Frau Simone Heil und Herr Thomas Germer haben viel Zeit und Energie in die „technische“ Erstellung des Handbuches gesteckt. 


\section{Inhaltsverzeichnis}

Vorwort der Herausgeber ................................................................................ V

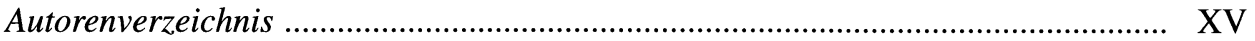

\section{Erster Teil}

\section{Industrielles Beschaffungsmanagement und Unternehmensführung}

\section{Lutz Kaufmann}

Purchasing and Supply Management - A Conceptual Framework

James Brian Quinn

Core-Competency-with-Outsourcing Strategies in Innovative Companies

Gerhard Schuff

Entwicklungsperspektiven für die Beschaffung in der Weltautomobilindustrie

Klaus Wangerin/Karen Chappell

„Globale Lokalisierung“-

Auswirkung und Umsetzung in der Beschaffung bei SONY in Europa

David N. Burt/Stephen Starling

World Class Supply Management

John Ramsay

Can Purchasing Have a Strategic Role in the Modern Firm?

Andreas Otto/Herbert Kotzab

Ziel erreicht? Sechs Perspektiven zur Ermittlung des Erfolgsbeitrags

des Supply Chain Management 


\section{Zweiter Teil}

\section{Strategische Ausrichtung des}

industriellen Beschaffungsmanagements

Thomas Freudenberg

Zulieferstrukturen im 21. Jahrhundert

Gerd Aberle/Alexander Eisenkopf

Wettbewerbspolitische Probleme innovativer Beschaffungskonzeptionen

Christian Homburg

Bestimmung der optimalen Lieferantenzahl für Beschaffungsobjekte:

Konzeptionelle Überlegungen und empirische Befunde

Ulli Arnold

Global Sourcing: Strategiedimensionen und Strukturanalyse

Mark Goh/Geok Theng Lau

International Procurement Offices in Asia

\section{Masaaki Kotabe}

Global Sourcing Strategy and Sustainability of Core Competencies:

Fundamental Differences between U.S. and Japanese Companies

Michael Eßig

Cooperative Sourcing

Ronald Bogaschewsky/Roland Rollberg

Produktionssynchrone Zulieferungskonzepte

Andreas R.Voegele/Roland Schwientek

Purchasing EmPowerment - Bestleistungen im Einkauf

Udo Jung/Siegfried Kiese/Alexander Tourneau

Strategic Procurement Initiative - How to Make it Happen 


\section{Dritter Teil}

\section{Zusammenspiel von Beschaffung und funktionalen Unternehmensbereichen}

Peter Wolters

Forward Sourcing - Entwicklungsbegleitende Lieferantenauswahl

Walter Kuemmerle

Global R\&D Strategies of Downstream Firms and the Role of Suppliers

Klaus Bellmann

Produktion und Beschaffung -

Management einer innerbetrieblichen Schnittstelle

Helmut Baumgarten/Inga-Lena Darkow

Versorgungsmanagement - Integration von Beschaffung und Logistik

Wolfgang Stölzle/Karin Gareis

Konzepte der Beschaffungslogistik -

Anforderungen und Gestaltungsalternativen

Armin Töpfer

Qualitätsmanagement-Konzepte bei veränderten Beschaffungsstrategien

Bernhard Nagel

Produkthaftung im Produktionsverbund:

Neue faktische und rechtliche Produkthaftungsrisiken

Thomas Dyllick/Jost Hamschmidt

Beschaffung und Umweltmanagement

Karl-Heinz Sebastian/Ralph Niederdrenk

Beschaffung und Verkauf - Von der Konfrontation zur Kooperation

Richard L. Pinkerton

The Evolution of Purchasing to Supply Chain Management 


\section{Vierter Teil}

\section{Innovative Instrumente für die industrielle Beschaffung}

Thomas Reichmann/Monika Palloks-Kahlen

Make-or-Buy-Kalkulationen im modernen Beschaffungsmanagement

Horst Wildemann

Das Konzept der Einkaufspotentialanalyse:

Bausteine und Umsetzungsstrategien

Günther R. Reinelt

Multimediale Beschaffungsmarktforschung

Oliver Fröhling/Martin G. Nonnenmacher

Purchasing Portfolio Analysis in the Future Market Environment

Johannes Rudnitzki

TANDEM - Die Lieferantenkooperation von DaimlerChrysler

Rainer Hoffmann/Hans-Joachim Lumbe

Lieferantenbewertung bei der Siemens AG -

Grundlage für das Lieferantenmanagement

Lisa M. Ellram

Total Cost of Ownership

Dietger Burghardt/Thomas Germer/Stefan Sippel

Flugzeugstandardisierung und Beschaffungsmanagement

der Deutschen Lufthansa AG

Jochen R. Pampel

Instrumente für das kooperationsbezogene Controlling von Produktions- und Transaktionskosten in der Supply Chain

Werner Seidenschwarz

Target Costing und Zuliefererintegration

Georg Möller/Klaus Möller

Konstruktionsbegleitendes Supply Chain Controlling mit prozeßorientiertem

Kostenmanagement

Bernadette Hausmann/Lutz Kaufmann

Re-engineering Supply Chain Management Activities for High Performance -

Process, Tools, and Results 


\section{Fünfter Teil}

Gestaltung von Organisationsstrukturen

und informationstechnologischen Systemen für die industrielle Beschaffung

Andrew Swan/Philip L. Carter/ Robert M. Monczka/Craig R. Carter

Coming into Focus: Using the Lens of Economic Value to Clarify the Impact of

B2B E-Marketplaces

Carlo Mackrodt/Henrich Dahm

Spreading trust - the glue for B2B eCommerce

797

Wolfgang Buchholz

Value Layer Procurement - Beschaffung als eigenständiges Geschäftsmodell

Michael Freienstein/René Petri/René Müller

Procurement Service Provider Development - Die Entwicklung des Zentraleinkaufs der Hoechst AG zu einem ,long-term value proposition“-Provider

Klaus Bellmann/Frank Himpel

Management von Beschaffungsnetzwerken

Wilfried Krüger/Christian Rohm/Christian Homp

Beschaffung im Fokus des Kernkompetenz-Managements

Harald Hungenberg

Organisation der Beschaffung in international tätigen Konzernen 901

Christof Weinhardt/Ralf Krause/Sven Herchenhein

Informationstechnologische Perspektiven für die Beschaffung

Heinrich Orths/Ute-Marie Ruland

Die Purchasing-Card als Rechnungssystem von elektronischen Marktplätzen 


\section{Sechster Teil}

\section{Gestaltung des Personalmanagements}

für die industrielle Beschaffung

Udo Koppelmann

Beschaffung als Forschungs- und Lehrzweig

Holger Hildebrandt

Der BME als Know-how-Drehscheibe des Beschaffungsmanagements

Jürgen Scherer

Personalkonzepte für die Beschaffung bei der Henkel KGaA

Christian Scholz

Vergütung und Entwicklung:

Motivationskonzepte für das Beschaffungsmanagement

Ralph Lindner

Aufgabenprofile und Gehaltsstrukturen im Einkauf in Deutschland 1005

Michael P. Zeuch

Beschaffung und Ethik

Ulrich Krystek

Beschaffung und Vertrauen 1039

\section{Ausblick}

Dietger Hahn

Problemfelder des Supply Chain Management 1061

Stichwortverzeichnis 


\section{Autorenverzeichnis}

Aberle, Gerd

Arnold, Ulli

Baumgarten, Helmut

Bellmann, Klaus

Bogaschewsky, Ronald

Buchholz, Wolfgang

Burghardt, Dietger

Burt, David N.

Carter, Craig $R$.
Prof. Dr., Inhaber des Lehrstuhls für Volkswirtschaftslehre, insbesondere Transportwirtschaft sowie Preis und Wettbewerb an der Justus-Liebig-Universität Gießen.

Prof. Dr. Dr. h.c., Inhaber des Lehrstuhls für Investitionsgütermarketing und Beschaffungsmanagement der Universität Stuttgart.

Prof. Dr.-Ing., Leiter des Bereichs Logistik und Direktor des Instituts für Technologie und Management an der TU Berlin.

Prof. Dr., Inhaber des Lehrstuhls für ABWL und Produktionswirtschaft sowie Direktor des Center for Market-Oriented Product and Production Management (CMPP) der JohannesGutenberg-Universität Mainz.

Prof. Dr., Inhaber des Lehrstuhls für Betriebswirtschaftslehre und Industriebetriebslehre, Julius-Maximilians-Universität Würzburg.

Dr., Gesellschafter und Management Berater bei eic-partner.

Dipl.-Ing., Leiter Strategischer Einkauf der Lufthansa Technik AG, Hamburg.

Professor, Ph.D., is currently the Director of the University of San Diego's undergraduate and graduate programs in procurement and supply management. $\mathrm{He}$ is founder and director of USD's Supply Chain Management Institute, and of the Strategic Supply Management Forum.

Ph.D., Assistant Professor of International Supply Chain Management, The Robert H. Smith School of Business, University of Maryland. 
Carter, Philip L.

Chappell, Karen

Dahm, Henrich

Darkow, Inga-Lena

Dyllick, Thomas

Eisenkopf, Alexander

Ellram, Lisa M.

Eßig, Michael

Freienstein, Michael

Freudenberg, Thomas

Fröhling, Oliver

Gareis, Karin
Ph.D., Executive Director, CAPS Research and Harold E. Fearon Eminent Scholar Chair of Purchasing, College of Business, Arizona State University.

MBA, zum Zeitpunkt der Erstellung des Artikels Group Leader for Procurement Planning and Systems, Sony Personal and Mobile Communications Europe.

Magister Artium, Dipl.-Betriebswirt (BA), Director Business Development, DCI AG.

Dipl.-Ing., wissenschaftliche Mitarbeiterin am Bereich Logistik der TU Berlin bei Prof. Dr.-Ing. H. Baumgarten.

Prof. Dr., Inhaber des Lehrstuhls für BWL mit besonderer Berücksichtigung des Umweltmanagements und Direktor des Instituts für Wirtschaft und Ökologie (IWÖ-HSG) der Universität St. Gallen.

PD Dr., wissenschaftlicher Assistent am Fachbereich Wirtschaftswissenschaften der JustusLiebig-Universität Gießen.

Ph.D., C.P.M., CPA, C.M.A., Associate Professor of Supply Chain Management at the College of Business Administration at Arizona State University (ASU) in Tempe, AZ.

Dr., Habilitand, wissenschaftlicher Mitarbeiter bei Prof. Dr. Dr. h.c. Ulli Arnold an der Universität Stuttgart.

Executive Vice President der ICGCommerce Europe.

Dr., Innomotive AG, München.

Dr., Senior Manager, Cap Gemini Ernst \& Young Deutschland GmbH, Düsseldorf.

Wissenschaftliche Mitarbeiterin am Fachgebiet Unternehmensführung an der Technischen Universität Darmstadt. 
Germer, Thomas

Goh, Mark

Hahn, Dietger

Hamschmidt, Jost

Hausmann, Bernadette

Herchenhein, Sven

Hildebrandt, Holger

Himpel, Frank

Hoffmann, Rainer
Dipl.-Kfm., wissenschaftlicher Mitarbeiter und Doktorand am Herbert-Quandt-Stiftungslehrstuhl für Betriebswirtschaftslehre, insbesondere Internationales Management, der WHU - Otto Beisheim Graduate School of Management in Vallendar/Koblenz.

Ph.D., currently an Associate Director with the Logistics Institute-Asia Pacific and an Associate Professor in the NUS Business School.

Prof. (em.) Dr. Dr. h.c. Dr.-Ing. E.h., zum Zeitpunkt der Erstellung des Handbuches Inhaber des Lehrstuhls für Industrielles Management und Controlling an der JustusLiebig-Universität Gießen sowie Honorarprofessor an der TU Berlin für strategisches Management. Geschäftsführender Leiter des Instituts für Unternehmungsplanung (IUP), Gießen/Berlin.

Dipl-Oec., wissenschaftlicher Assistent am Lehrstuhl für BWL mit besonderer Berücksichtigung des Umweltmanagements und am Institut für Wirtschaft und Ökologie (IWÖHSG) der Universität St. Gallen.

Dr., MBA, Head of Corporate Supply Chain Management and Strategic Tire Technology at Continental AG, Hannover, Germany.

Dipl.-Kfm, Dipl.-Inform. (FH), Vorstand der trading fair AG, Gießen.

Dr., Hauptgeschäftsführer des Bundesverbandes Materialwirtschaft, Einkauf und Logistik e. V. (BME) in Frankfurt/Main.

Wissenschaftlicher Mitarbeiter am Lehrstuhl für ABWL und Produktionswirtschaft sowie Doktorand am Center for Market-Oriented Product and Production Management (CMPP) der Johannes-Gutenberg-Universität Mainz.

Geschäftsführender Partner der Hoffmann \& Zachau, Unternehmensberatung. 
Homburg, Christian

Homp, Christian

Hungenberg, Harald

Jung, Udo

Kaufmann, Lutz

Kiese, Siegfried

Koppelmann, Udo

Kotabe, Masaaki

Kotzab, Herbert

Krause, Ralf

Krüger, Wilfried
Prof. Dr., Direktor des Instituts für Marktorientierte Unternehmensführung an der Universität Mannheim; Vorsitzender des Wissenschaftlichen Beirats der Prof. Homburg \& Partner $\mathrm{GmbH}$, einer international tätigen Management Beratung.

Dr., Projektleiter Management Systems bei SCHOTT Glas, Mainz.

Prof. Dr., Inhaber des Lehrstuhls für Allgemeine Betriebswirtschaftslehre und Unternehmensführung der Friedrich-AlexanderUniversität Erlangen-Nürnberg.

Dr., Vice President at the Frankfurt Office of The Boston Consulting Group and Head of the German Operational Effectiveness Practice.

Prof. Dr., MBA, Inhaber des Herbert-QuandtStiftungslehrstuhls für Betriebswirtschaftslehre, insbesondere Internationales Management, an der WHU - Otto Beisheim Graduate School of Management in Vallendar/Koblenz.

Dr., Head of Material Management (Procurement \& Logistics) of Wacker Chemie GmbH.

Prof. Dr., Direktor des Seminars für Allgemeine Betriebswirtschaftslehre, Beschaffung und Produktpolitik der Universität zu Köln.

Ph.D., The Washburn Chair of International Business and Marketing at the Institute of Global Management Studies at Temple University, Philadelphia, PA.

Dr., Associate Professor, Department of Operations Management, Copenhagen Business School.

Dipl.-Kfm., Analyst bei Union Investment, Frankfurt/Main.

Prof. Dr., Inhaber des Lehrstuhls für Organisation, Unternehmungsführung und Personalwirtschaft der Justus-Liebig-Universität Gießen. 
Krystek, Ulrich

Kuemmerle, Walter

Lindner, Ralph

Lumbe, Hans-Joachim

Mackrodt, Carlo

Möller, Georg

Möller, Klaus

Monczka, Robert M.

Müller, René

Nagel, Bernhard

Niederdrenk, Ralph

Nonnenmacher, Martin G.
Prof. Dr., Internationale Fachhochschule Worms, Gastprofessor an der TU Berlin, Fachbereich Wirtschaft und Management, Vertreter des Lehrgebietes Unternehmensrechnung und Controlling seit 1996.

D.B.A., Associate Professor of Business Administration, Graduate School of Business Administration, Harvard University, Cambridge, MA.

Dipl.-Ing., Verlagsleiter des Konradin-Verlags, Leinfelden.

Dipl.-Kfm., Dipl.-Ing. (FH), Vice President Procurement Development, Siemens AG, München.

Dr.-Ing., Vice President Shared Service Organization Europe, Henkel KGaA, Düsseldorf.

Dipl.-Wirtsch.-Ing., Leiter Materialwirtschaft und verantwortlich für das Supply Chain Management der Wittenstein AG, Igersheim.

Dipl.-Wirtsch.-Ing., wissenschaftlicher Mitarbeiter am Lehrstuhl Controlling von Prof. Horváth an der Universität Stuttgart sowie Projektleiter verschiedener Forschungsprojekte mit der Industrie im Bereich der Antriebstechnik.

Ph.D., Director, Project 10X, CAPS Research and Professor of Supply Chain Management, College of Business, Arizona State University.

Consultant der ICGCommerce Europe.

Prof. Dr., Inhaber des Lehrstuhls für Wirtschaftsrecht am Fachbereich Angewandte Sozialwissenschaften, Rechtswissenschaft der Universität Gesamthochschule Kassel.

Dipl.-Kfm., Consultant bei der SIMON KUCHER \& PARTNERS GmbH, Bonn.

Prof. Dr., Partner of the Arthur Andersen Business Consulting GmbH, Stuttgart. 
Orths, Heinrich

Otto, Andreas

Palloks-Kahlen, Monika

Pampel, Jochen R.

Petri, René

Pinkerton, Richard L.

Quinn, James Brian

Ramsay, John

Reichmann, Thomas

Reinelt, Günther R.

Rohm, Christian

Rollberg, Roland
Supply Manager ABB Calor Emag Mittelspannung $\mathrm{GmbH}$, Ratingen.

Dr. habil., Product Manager Fulfillment, GBU Supply Chain Management, SAP AG.

Dr., wissenschaftliche Assistentin am Lehrstuhl für Controlling und Unternehmensrechnung der Universität Dortmund.

PD Dr., Privatdozent an der Wirtschafts- und Sozialwissenschaftlichen Fakultät der Universität Erlangen-Nürnberg und Lehrbeauftragter des Hasso-Plattner-Instituts für Softwaresystemtechnik an der Universität Potsdam.

Director Program Management der ICGCommerce Europe.

Ph.D., C.P.M., Chair and Professor of Marketing and Logistics, The Sid Craig School of Business, California State University, Fresno, CA.

Ph.D., William and Josephine Buchanan Professor of Management, Emeritus, The Amos Tuck School at Dartmouth College, Hanover, NH.

Senior Lecturer, Economics Division, Staffordshire University Business School.

Prof. Dr., Inhaber des Lehrstuhls für Controlling und Unternehmensrechnung der Universität Dortmund.

Dr.-Ing., Leiter Zentralbereich Einkauf der Miele \& Cie GmbH \& Co., Gütersloh.

Dr., Geschäftsführer der STI Gustav Stabernack GmbH Unternehmensgruppe, Lauterbach, und verantwortlich für das Ressort Finanzen und Controlling.

Prof. Dr. habil., Inhaber des Lehrstuhls für Allgemeine Betriebswirtschaftslehre und Produktionswirtschaft der Ernst-Moritz-ArndtUniversität Greifswald. 
Rudnitzki, Johannes

Ruland, Ute-Marie

Scherer, Jürgen

Scholz, Christian

Schuff, Gerhard

Schwientek, Roland

Sebastian, Karl-Heinz

Seidenschwarz, Werner

Sippel, Stefan

Starling, Stephen

Stölzle, Wolfgang
Mitglied des Direktoriums der DaimlerChrysler AG und Leiter des Materialeinkaufs MB-PKW der DaimlerChrysler AG.

Dipl.-Betriebswirtin (FH), Supply Management ABB Calor Emag Mittelspannung $\mathrm{GmbH}$, Ratingen.

Dr., Vice President Corporate Key Account Management, Cognis Deutschland $\mathrm{GmbH} \&$ Co. KG, Düsseldorf. Zum Zeitpunkt der Erstellung des Artikels war er bei der Henkel $\mathrm{KGaA}$ tätig.

Prof. Dr., Inhaber des Lehrstuhls für Betriebswirtschaftslehre, insbesondere Organisation, Personal- und Informationsmanagement der Universität des Saarlandes, Saarbrücken.

Dr., Leiter der Materialwirtschaft der BMW Group.

Associate Partner im internationalen Competence Center Operations Strategy, Roland Berger - Strategy Consultants, Stuttgart.

Dr., Gründungsgesellschafter und Partner der SIMON KUCHER \& PARTNERS GmbH, Bonn.

Dr., Vertreter des Lehrstuhls Controlling und Unternehmensrechnung der Wirtschaftswissenschaftlichen Fakultät i. G. der Technischen Universität München und geschäftsführender Gesellschafter der Seidenschwarz \& Comp. GmbH, Starnberg.

Dipl.-Kfm., Director Aircraft Purchasing, Deutsche Lufthansa AG, Frankfurt.

Ph.D., Associate Professor at the faculty of the Supply Chain Management Institute at the University of San Diego.

Prof. Dr., Inhaber des Lehrstuhls für Betriebswirtschaftslehre mit dem Schwerpunkt Logistik und Verkehrsbetriebslehre an der Gerhard-Mercator-Universität Duisburg. 
Swan, Andrew

Theng Lau, Geok

Töpfer, Armin

Tourneau, Alexander

Voegele, Andreas $R$.

Wangerin, Klaus

Weinhardt, Christof

Wildemann, Horst

Wolters, Peter

Zeuch, Michael P.
Knowledge Manager, McKinsey \& Company, Chicago.

Ph.D., currently an Associate Professor in the Department of Marketing, National University of Singapore.

Prof. Dr., Inhaber des Lehrstuhls für Marktorientierte Unternehmensführung an der TU Dresden und Leiter der Forschungsgruppe Management + Marketing in Kassel.

Dr., Manager at the Hamburg Office of The Boston Consulting Group and Topic Leader for e-Procurement.

Dr.-Ing., Senior Partner und Leiter des internationalen Competence Center Operations Strategy, Roland Berger - Strategy Consultants, Stuttgart.

Direktor, European Procurement Division, Sony International (Europe) $\mathrm{GmbH}$.

Prof. Dr., Inhaber des Lehrstuhls für Informationsbetriebswirtschaftslehre an der Universität Karlsruhe $(\mathrm{TH})$.

Prof. Dr. Dr. habil., Inhaber des Lehrstuhls für Betriebswirtschaftslehre mit Schwerpunkt Logistik an der Technischen Universität München.

Dipl.-Ing., Dipl.-Oec., Konzernbereichsleiter für den Bereich Global/Forward Sourcing und Internationale Projektsteuerung bei der Volkswagen AG, Wolfsburg.

Prof. Dr.-Ing., Fachhochschule WürzburgSchweinfurt, Fachbereich Wirtschaftsingenieurwesen und Betriebswirtschaft; Mitglied des Bundesvorstandes BME; Unternehmensberater und Leiter des Center of Excellence für Prozess-Stabilisierung kleiner und mittlerer Zuliefer-Unternehmen (international). 